(C) 2021 IEEE. Personal use of this material is permitted. Permission from IEEE must be obtained for all other uses, in any current or future media, including reprinting/republishing this material for advertising or promotional purposes, creating new collective works, for resale or redistribution to servers or lists, or reuse of any copyrighted component of this work in other works. 


\section{Limitations in Applying the Existing LISN Topologies for Low Frequency Conducted Emission Measurements and Possible Solution}

\author{
Lu Wan \\ $D E I B$ \\ Politecnico di Milano \\ Milan, Italy \\ lu.wan@polimi.it
}

\author{
Arun Khilnani \\ GGIEMR \\ University of Nottingham \\ Nottingham, The UK \\ arun.khilnani@nottingham.ac.uk
}

Sergio Pignari

DEIB
Giordano Spadacini DEIB

Politecnico di Milano Milan, Italy

\author{
Politecnico di Milano \\ Milan, Italy
}

o.pignari@po

\author{
Abduselam Hamid \\ $D E I B$ \\ Politecnico di Milano \\ Milan, Italy \\ abduselamhamid.beshir@polimi.it
}

\author{
Flavia Grassi \\ DEIB \\ Politecnico di Milano \\ Milan, Italy \\ flavia.grassi@polimi.it
}

University of Nottingham

Nottingham, The UK

\author{
David Thomas \\ GGIEMR \\ University of Nottingham \\ Nottingham, The UK
}

\begin{abstract}
The use of power electronic converters to interface renewable sources and intelligent loads to electricity distribution systems is increasing at a rapid rate as they bring flexibility and control to the system. However, they also bring an increased level of conducted emissions (CE) to the system due to their switching behaviour - usually at a few tens of kilohertz. The increased emissions are seen particularly in the low-frequency range (2-150 $\mathrm{kHz}$ ) and it may possibly impair the operation of information and communication technology (ICT) equipment connected to the same system. It is therefore essential to assure accurate measurement of low frequency emissions from a particular piece of equipment, to ensure it meets standards for electromagnetic compatibility (EMC). According to EMC standards, CE are usually measured by using a Line Impedance Stabilization Network (LISN). However, the standard LISN bandwidth does not fully cover this low-frequency range $(2-150 \mathrm{kHz})$, resulting in inaccurate measurement and poor repeatability. This paper examines this issue, by investigating the limitations in using the CISPR 16-1-2 LISN topology for CE measurement at low frequency in a grid-tied inverter system, and by proposing a possible solution, which requires adding extra components to the LISN circuit.
\end{abstract}

Index Terms-LISN, conducted emissions, low frequency, electromagnetic interference

\section{INTRODUCTION}

The increasing use of renewable energy sources such as photovoltaic panels and wind generators had led to a significant increase in the number of power electronic converters connected to the electricity grid, and this in turn has led to an increase in the electromagnetic interference (EMI) in modern power systems, [1]-[3]. The EMI between 2-150 kHz is especially accentuated, since the switching frequency of high power inverters is still limited to several $\mathrm{kHz}$ [4], [5]. However, this frequency range is not covered either by Power Quality or EMC standards, where Power Quality ranges from
$50 \mathrm{~Hz}$ to the 40th harmonics $(2 \mathrm{kHz})$ only, and EMC is considered from $9 \mathrm{kHz}$ or $150 \mathrm{kHz}$ up to $30 \mathrm{MHz}$ [6][8]. This lack of regulation can result in compatibility issues between renewable energy systems and ICT devices, and also introduces problems into the measurement of emissions. For instance, the operating frequency of a LISN typically specified in EMC Standards does not fully cover the $2-150 \mathrm{kHz}$ range (the frequency range for CISPR 22 and CISPR 16 are from $150 \mathrm{kHz}-30 \mathrm{MHz}$ and from $9 \mathrm{kHz}-30 \mathrm{MHz}$, respectively).

Within its specified frequency range, a LISN can provide a stable input impedance for CE measurement in spite of variations of the mains impedance. The LISN acts as Low Pass Filter (LPF) to ensure that the power (AC or DC) is transferred to the load device yet uncoupling the measuring receiver from the high-frequency noise coming from the main utility supply. In this way, only the CE exiting the device under test (DUT) are measured at the LISN over the specified frequency range [9]. However, for frequencies ouside the LISN bandwidth (here frequencies below $9-150 \mathrm{kHz}$ are of interest), the aforesaid LISN features are not guaranteed. Hence, applying existing LISNs to measure CE levels outside this frequency range may lead to unpredictable and unrepeatable results. CE measurement below $9 \mathrm{kHz}$ has not received much attention so far in the wider literature, and well designed LISN devices for this frequency range are still not commercially available. This paper aims to analyse the shortcomings in existing LISN topologies when used for measuring conducted emissions from power converters at these low frequencies and also to propose a possible solution for LISN design.

\section{Limitations OF Existing LISNS}

The first limitation is that the LISN impedance can exhibit unexpected deviations depending on the mains impedance be- 

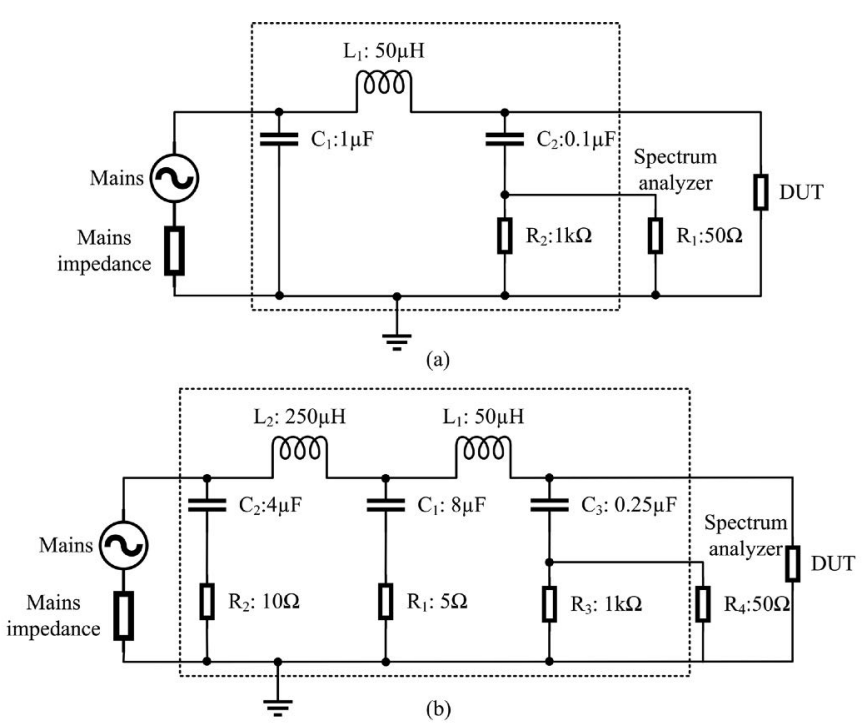

Fig. 1: LISN topologies specified in CISPR 16-1-2: (a)50 $\mu \mathrm{H}$ || $50 \Omega$ and (b) $(50 \mu \mathrm{H}+5 \Omega) \| 50 \Omega$.

low the specified operating bandwidth. For instance, the EMC Standards, such as CISPR 16, CISPR 22 and FCC, specify the LISN topology in Fig. 1(a) for the use in Band B (150 kHz-30 $\mathrm{MHz}$ ). The LISN impedance (both magnitude and phase) seen by the DUT is defined as a $50 \mu \mathrm{H}$ inductor in parallel with a $50 \Omega$ resistor: $(50 \mu \mathrm{H} \| 50 \Omega$ plotted as Std. Band B in Fig. 2). The CISPR 16-1-2 standard allows $20 \%$ tolerance for the magnitude and $\pm 11.5^{\circ}$ for the phase. However, if the mains impedance changes, the overall impedance seen from the DUT outlets may exhibit significant variations below $150 \mathrm{kHz}$ (see Fig.2, where this impedance is plotted for different values of the impedance of the mains). Similarly, the topology in Fig. 1(b) is used in Band A (9 kHz-150 kHz), where the LISN impedance is defined as $(50 \mu \mathrm{H}+5 \Omega) \| 50 \Omega$ (Std. Band A in Fig. 3). This topology usually can satisfy the requirement in Band B as well, which makes it usable for both bands, i.e., from $9 \mathrm{kHz}$ to $30 \mathrm{MHz}$. Similar to the $50 \mu \mathrm{H} \| 50$ $\Omega$ topology, this one also cannot provide stable impedance against a varying mains impedance below $9 \mathrm{kHz}$.

The unstable impedance at low frequency can be explained by inspecting the LISN topology. For instance, in Fig. 1 (b), on the one hand, the $(50 \mu \mathrm{H}+5 \Omega) \| 50 \Omega$ LISN impedance is defined by $L_{1}, C_{1}, R_{1}, R_{3}$ and $R_{4}$, which exhibits approximately response as $(50 \mu \mathrm{H}+5 \Omega) \| 50 \Omega$ at high frequency. However, at low frequency, the $0.25 \mu \mathrm{F}$ capacitance of $C_{3}$ does not show a negligible impedance. On the other hand, the parameters $L_{2}, C_{2}$ and $R_{2}$ works as a LPF to suppress the spurious mains signals and to insulate the mains impedance variations. However, this LPF does not sufficiently work well at low frequency.

The other two limitations at low frequency are: 1) insufficient isolation between the mains port and the receiver port, which can allow spurious signal to influence $\mathrm{CE}$ measurement and, 2) the CE from the DUT port to the receiver port are

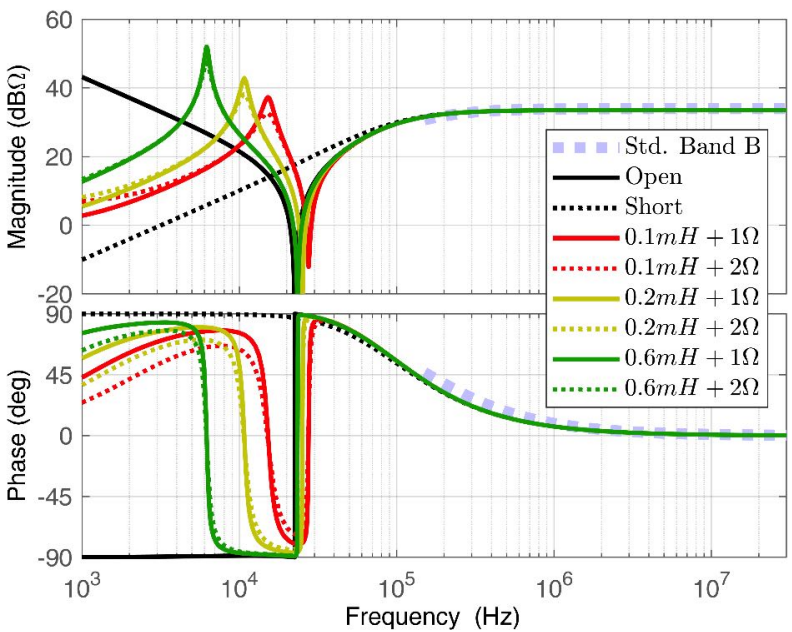

Fig. 2: Impedances of $50 \mu \mathrm{H} \| 50 \Omega$ LISN under different power grid impedance.

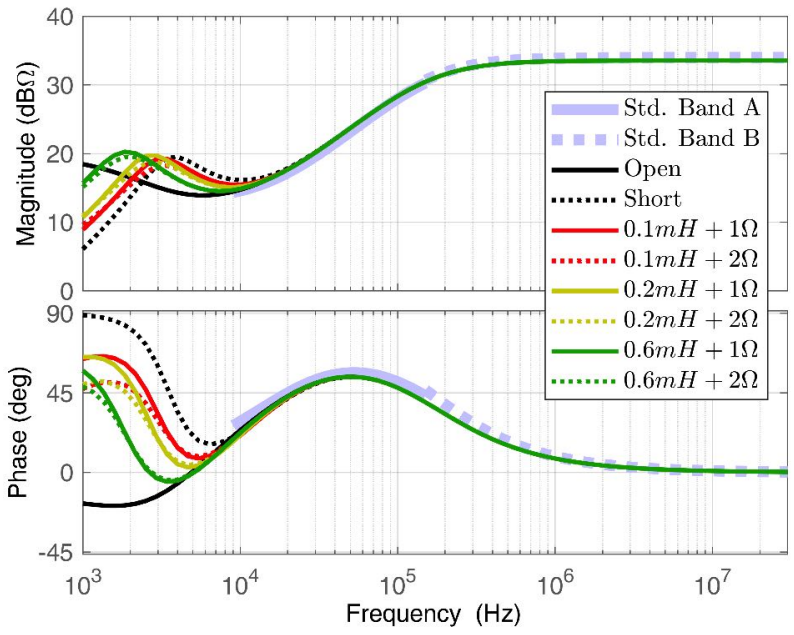

Fig. 3: Impedances of $(50 \mu \mathrm{H}+5 \Omega) \| 50 \Omega$ LISN under different power grid impedance.

attenuated significantly, which limits $\mathrm{CE}$ assessment at low frequency. These two limitations can be associated with the Decoupling Factors (DF) and the Voltage Division Factor (VDF) defined in CISPR 16-1-2 [9]. First, a minimum isolation (DF) between each mains terminal and the LISN receiver port for a given termination (specified as $50 \Omega$ resistor in CISPR161-2) of DUT port shall be met, so to ensure the following two functions of the LISN at any test frequency: 1) isolation of the unknown mains impedance to prevent its influence on CE measurement, and 2) suppression of unwanted spurious signals existing on the mains side. The minimum $40 \mathrm{~dB}$ DF is required above $50 \mathrm{kHz}$ and requirement from $9 \mathrm{kHz}$ to 50 $\mathrm{kHz}$ rises linearly with the logarithm of frequency (as plotted in Fig. 4 in dot blue lines). Second, the VDF is defined as the voltage ratio between DUT port and the LISN receiver port (terminated with a $50 \Omega$ load), which needs to be taken into account when CE are measured. In Fig. 4, both factors of 


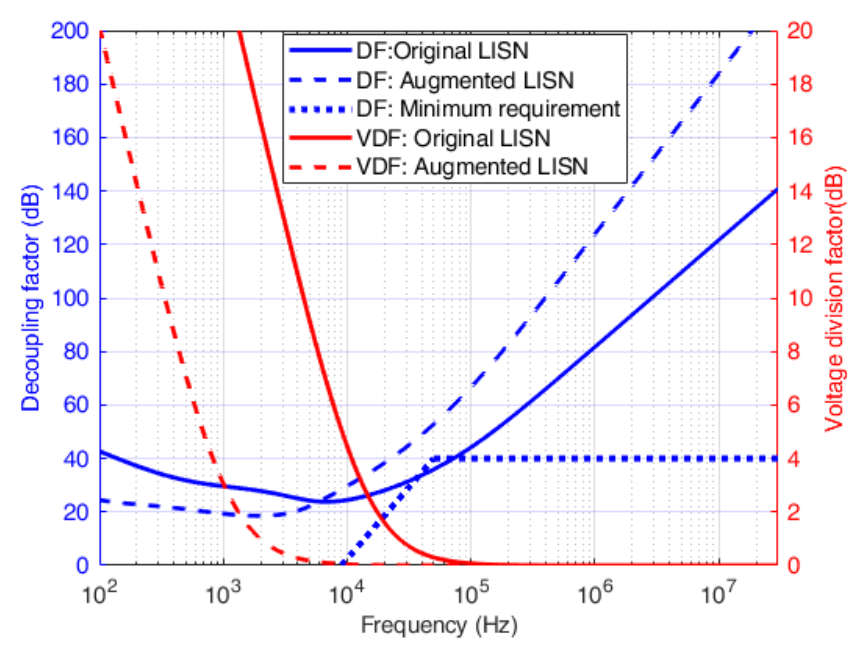

Fig. 4: Decoupling factor and voltage division factor of $(50 \mu \mathrm{H}+5 \Omega) \| 50 \Omega$ LISN and augmented LISN.

the original $(50 \mu \mathrm{H}+5 \Omega) \| 50 \Omega$ LISN are plotted (blue and red curve). Fig. 4 highlights the necessity to increase the DF and reduce the VDF at low frequency, in order to suppress further the spurious noise at low frequency from the mains side and better decouple the disturbance voltage from DUT port to receiver port below $9 \mathrm{kHz}$, respectively.

\section{CE MEASUREMENT OF INVERTER SySTEM}

In order to investigate the aforesaid limitations in $\mathrm{CE}$ measurement, the virtual test setup shown in Fig. 5 was built in SPICE, wherein the DUT is the three-phase inverter system connected with a PV panel working at $5 \mathrm{kHz}$ switching frequency. The power grid is emulated using $50 \mathrm{~Hz}$ threephase voltage sources and the mains impedance as $0.2 \mathrm{mH}$ inductor in series with a $1 \Omega$ resistor. It is worth mentioning that both functional aspects and parasitic components are considered in the SPICE model. The frequency spectrum is evaluated through Fast Fourier Transforms from the timedomain simulation results.

In Fig. 6, the CE spectra obtained by using the two LISNs in Fig. 1 are compared from $2 \mathrm{kHz}$ to $1 \mathrm{MHz}$. It results that the evaluated CE above $70 \mathrm{kHz}$ are identical for both LISN topologies. Conversely, at low frequency, the CE evaluated by two LISNs show significant discrepancies. This phenomenon can be ascribed to the LISN impedance which is highly influenced by the mains impedance outside the LISN bandwidth, as shown in Fig. 2 and Fig. 3. It is worth mentioning that, although the $(50 \mu \mathrm{H}+5 \Omega) \| 50 \Omega$ LISN can be used to assess $\mathrm{CE}$ above $9 \mathrm{kHz}$, in this application, the fundamental switching frequency $(5 \mathrm{kHz})$ falls below the bandwidth, thus the obtained results at $5 \mathrm{kHz}$ cannot be regarded as valid due to its dependence on the test setups (mains impedance).

To illustrate the insufficient isolation provided by the LISN at low frequency, another similar test setup exploiting only $(50 \mu \mathrm{H}+5 \Omega) \| 50 \Omega$ LISN was simulated by SPICE in the presence and absence of spurious noise from the mains. For each mains phase, the disturbance contains 20 voltage sources

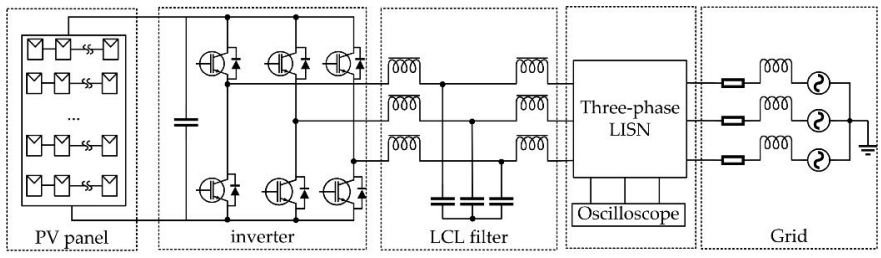

Fig. 5: Setups of CE measurement of grid-tied three-phase inverter system using existing LISN.

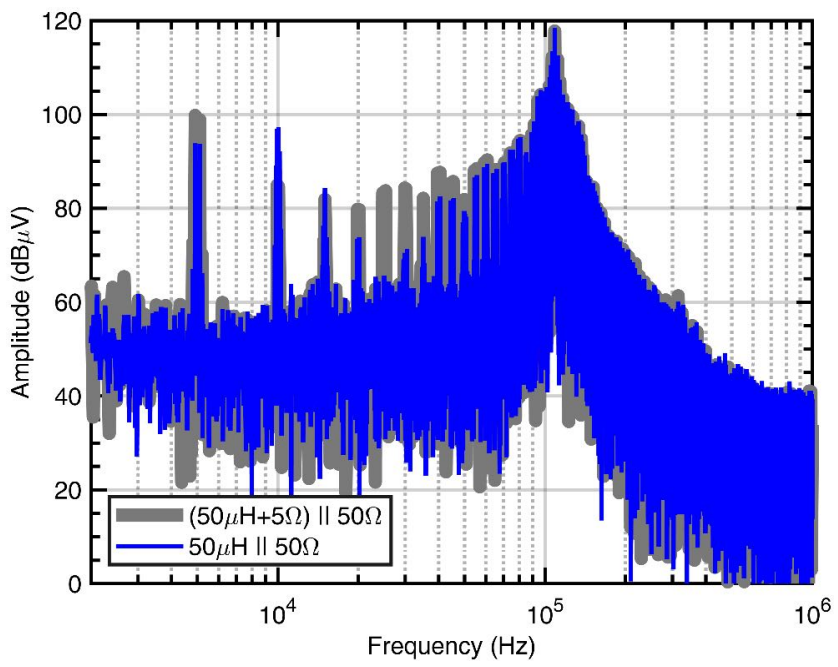

Fig. 6: CE of one phase of inverter system using $(50 \mu \mathrm{H}+5 \Omega)$ || $50 \Omega$ and $50 \mu \mathrm{H} \| 50 \Omega$ LISN.

with $0.1 \mathrm{~V}$ amplitude each in series, from $2.5 \mathrm{kHz}$ and its harmonics until $50 \mathrm{kHz}$. The measured $\mathrm{CE}$ in both cases are plotted in Fig. 7, the case with disturbance shows that the LISN cannot effectively suppress the mains noise below 20 $\mathrm{kHz}$, thus implying spurious emission peaks in the measured CE.

\section{Possible Solution}

To improve the characteristics of the $(50 \mu \mathrm{H}+5 \Omega) \| 50$ $\Omega$ LISN at low frequency three goals must be achieved: 1) keeping the LISN impedance stable at low frequency in spite of variations of the mains impedance, 2) increasing the DF further to suppress the noise from the mains, and 3) improving the coupling of CE at DUT side above certain frequency to the receiver port. To this end, two adapting breakout boxes containing a LPF and a high-pass filter (HPF), respectively, are attached at the mains side and output side of the LISN, respectively, as shown in Fig. 8.

The HPF breakout box has the cutoff frequency at $1 \mathrm{kHz}$ using $3.3 \mu \mathrm{F}$ capacitor $\left(C_{H P F}\right)$ and $1 \mathrm{k} \Omega$ resistor $\left(R_{H P F}\right)$, so as to have smaller VDF at low frequency (shown as red dash curve) in Fig. 4. Thus CE above $1 \mathrm{kHz}$ from the DUT can be recorded using this HPF breakout box. The attached LPF can not only mask the variations of the mains impedance at low frequency, but also increase the DF to suppress the spurious signals at mains side. For instance, using $400 \mu \mathrm{H}$ inductor 


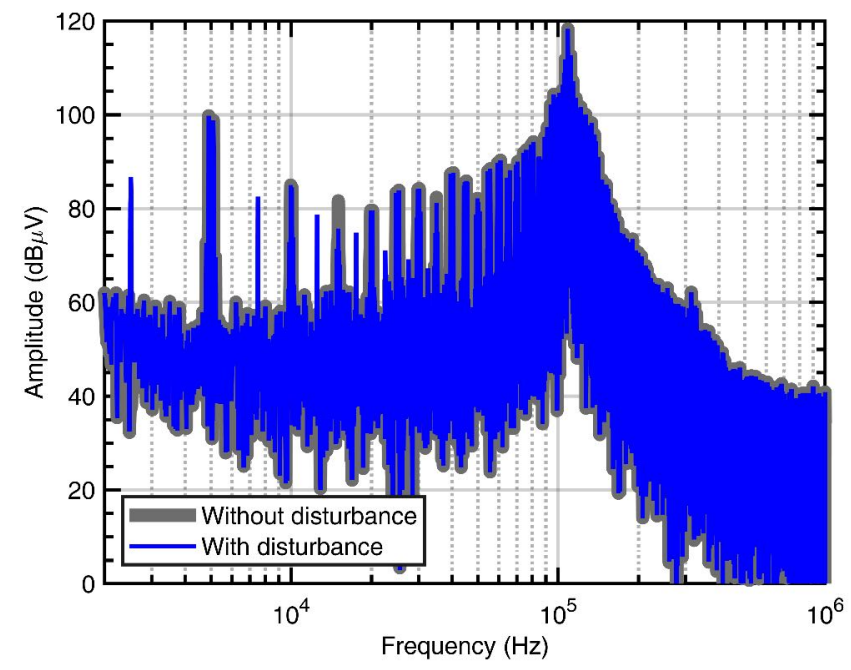

Fig. 7: CE measurement of one phase of inverter system using $(50 \mu \mathrm{H}+5 \Omega) \| 50 \Omega \mathrm{LISN}$ in the presence and absence of the disturbance from grid.

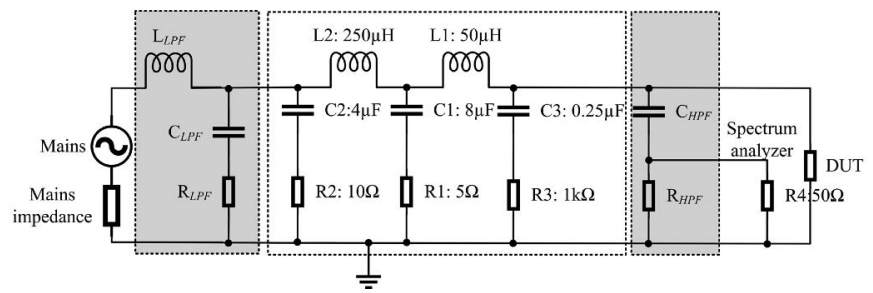

Fig. 8: Adapting LPF and HPF breakout boxes based on $(50 \mu \mathrm{H}+5 \Omega) \| 50 \Omega$ LISN.

$\left(L_{L P F}\right), 60 \mu \mathrm{F}$ capacitor $\left(C_{L P F}\right)$ and $5 \Omega$ resistor $\left(R_{L P F}\right)$ along with the aforesaid HPF breakout box for augmentation of the $(50 \mu \mathrm{H}+5 \Omega) \| 50 \Omega$ LISN, the characteristics and the aforementioned three limitations can be improved as shown in Fig.4 and Fig. 9. First, the augmented LISN impedance is stable above $2 \mathrm{kHz}$ in spite of variations of the mains impedance. The largest variation from $2-150 \mathrm{kHz}$ is less than $2 \mathrm{~dB} \Omega$ at $2 \mathrm{kHz}$ (except the open and short cases). Second, the DF is increased and VDF is decreased at low frequency compared with the original LISN. It is worth noting that by reducing further the cutoff frequency of the LPF breakout box, better characteristics can be achieved from $2 \mathrm{kHz}$. However, this action represents a tradeoff between better characteristics and other requirements. For instance, the bulky inductor in the LPF has a low self-resonance frequency due to the inherent parasitic capacitor, which limits the upper operating frequency of the LISN. Moreover, the leakage current of the capacitor in the LPF should be limited by using several capacitors in parallel and a resistor in series with each capacitor.

\section{CONCLUSions}

This paper has investigated the three main limitations of using traditional LISNs for CE measurement starting from 2 $\mathrm{kHz}$. These are 1) its unstable impedance under variations of

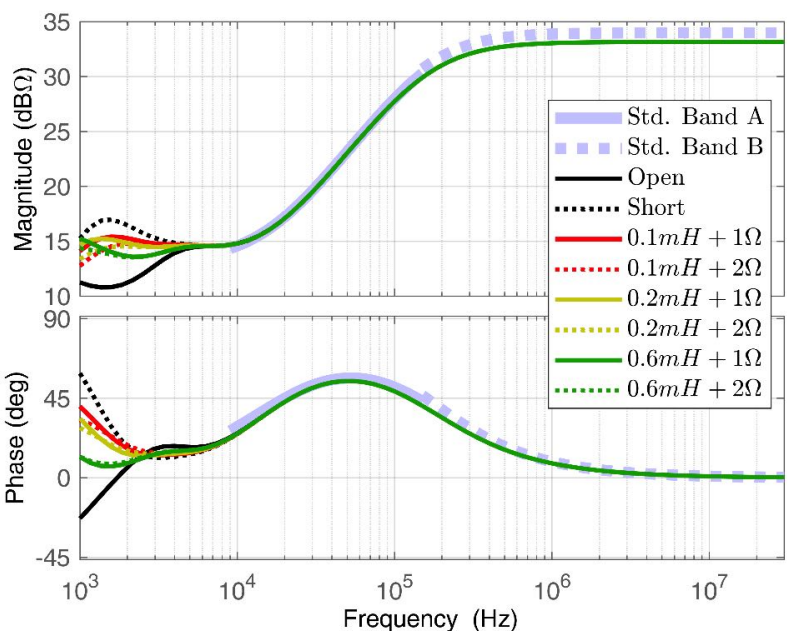

Fig. 9: Impedance of augmented $(50 \mu \mathrm{H}+5 \Omega) \| 50 \Omega$ LISN.

the supply utility impeance, b) its week provision for isolation between the utility and the DUT, and c) the poor coupling of the DUT to the receiver port at low frequencies. These limitations are illustrated by simulating the $\mathrm{CE}$ measurement in a grid-tied three-phase inverter system. Finally, a possible solution is proposed which adds circuit elements to the existing LISN topology to improve its low frequency performance.

\section{REFERENCES}

[1] R. Araneo, S. Lammens, M. Grossi, and S. Bertone, "Emc issues in high-power grid-connected photovoltaic plants," IEEE Transactions on Electromagnetic Compatibility, vol. 51, no. 3, pp. 639-648, 2009.

[2] G. Rietveld, D. Hoogenboom, and M. Acanski, "Conducted emi causing error readings of static electricity meters," in 2018 Conference on Precision Electromagnetic Measurements (CPEM 2018), 2018, pp. 1-2.

[3] J. B. Noshahr and B. M. Kalasar, "Evaluating emission and immunity of harmonics in the frequency range of $2-150 \mathrm{khz}$ caused by switching of static convertor in solar power plants," CIRED-Open Access Proceedings Journal, vol. 2017, no. 1, pp. 625-628, 2017.

[4] D. Darmawardana, S. Perera, D. Robinson, P. Ciufo, J. Meyer, M. Klatt, and U. Jayatunga, "Investigation of high frequency emissions (supraharmonics) from small, grid-tied, photovoltaic inverters of different topologies," in 2018 18th International Conference on Harmonics and Quality of Power (ICHQP), 2018, pp. 1-6.

[5] S. Rönnberg, M. Bollen, and A. Larsson, "Emission from small scale pv-installations on the low voltage grid," in The Renewable Energies and Power Quality Journal (RE\&PQJ), no. 12, 2014.

[6] S. K. Rönnberg, M. H. Bollen, H. Amaris, G. W. Chang, I. Y. Gu, Ł. H. Kocewiak, J. Meyer, M. Olofsson, P. F. Ribeiro, and J. Desmet, "On waveform distortion in the frequency range of $2 \mathrm{khz}-150 \mathrm{khz}$-review and research challenges," Electric Power Systems Research, vol. 150, pp. 1-10, 2017.

[7] S. Subhani, V. Cuk, and J. Coben, "A literature survey on power quality disturbances in the frequency range of 2-150 khz," in Proceedings of the International Conference on Renewable Energies and Power Quality (ICREPQ'14), Málaga, Spain, 2017, pp. 4-6.

[8] J. Meyer, V. Khokhlov, M. Klatt, J. Blum, C. Waniek, T. Wohlfahrt, and J. Myrzik, "Overview and classification of interferences in the frequency range 2-150 khz (supraharmonics)," in 2018 International Symposium on Power Electronics, Electrical Drives, Automation and Motion (SPEEDAM), 2018, pp. 165-170.

[9] CISPR16-1-2, "Specification for radio disturbance and immunity measuring apparatus and methods - Part 1-2: Radio disturbance and immunity measuring apparatus - Coupling devices for conducted disturbance measurements," International Electrotechnical Commission, Standard, 2014. 